TRANSACTIONS OF THE

AMERICAN MATHEMATICAL SOCIETY

Volume 353, Number 10, Pages 4203-4217

S 0002-9947(01)02850-1

Article electronically published on June 6, 2001

\title{
ON THE COMMUTATIVITY OF THE ALGEBRA OF INVARIANT DIFFERENTIAL OPERATORS ON CERTAIN NILPOTENT HOMOGENEOUS SPACES
}

\author{
HIDÉNORI FUJIWARA, GÉRARD LION, AND SALAH MEHDI
}

\begin{abstract}
Let $G$ be a simply connected connected real nilpotent Lie group with Lie algebra $\mathfrak{g}, H$ a connected closed subgroup of $G$ with Lie algebra $\mathfrak{h}$ and $\beta \in \mathfrak{h}^{*}$ satisfying $\beta([\mathfrak{h}, \mathfrak{h}])=\{0\}$. Let $\chi_{\beta}$ be the unitary character of $H$ with differential $2 \sqrt{-1} \pi \beta$ at the origin. Let $\tau \equiv \operatorname{Ind}_{H}^{G} \chi_{\beta}$ be the unitary representation of $G$ induced from the character $\chi_{\beta}$ of $H$. We consider the algebra $\mathcal{D}(G, H, \beta)$ of differential operators invariant under the action of $G$ on the bundle with basis $H \backslash G$ associated to these data. We consider the question of the equivalence between the commutativity of $\mathcal{D}(G, H, \beta)$ and the finite multiplicities of $\tau$. Corwin and Greenleaf proved that if $\tau$ is of finite multiplicities, this algebra is commutative. We show that the converse is true in many cases.
\end{abstract}

\section{Notations And FORMulation of The QUeStion}

Let $G$ be a simply connected connected real nilpotent Lie group with Lie algebra $\mathfrak{g}$ and $H$ a connected closed subgroup of $G$ with Lie algebra $\mathfrak{h}$. For $l \in \mathfrak{g}^{*}$, we denote by $\mathfrak{g}(l)$ the Lie algebra of the stabilizer $G(l)$ of $l$ under the co-adjoint action $A d^{*}$ of $G$ on $\mathfrak{g}^{*}$. For $\beta \in \mathfrak{h}^{*}$ satisfying $\beta([\mathfrak{h}, \mathfrak{h}])=\{0\}$, the homomorphism $\beta$ induces a character $\chi_{\beta}$ of $H$ with $2 \sqrt{-1} \pi \beta$ as differential at the origin. We then form the unitary induced representation $\tau \equiv \operatorname{Ind}_{H}^{G} \chi_{\beta}$ of $G$ in $\mathcal{H}_{\tau}$ realized, in the usual way, as the completion of a vector subspace of $C^{\infty}(G, H, \beta)$, namely the vector space of the $C^{\infty}$ complex functions $f$ on $G$ satisfying the following covariance relation:

$$
f(h g)=\chi_{\beta}(h) f(g) \forall h \in H \forall g \in G .
$$

The action of $G$ is given by right translations:

$$
\tau\left(g^{\prime}\right)(f)(g)=f\left(g g^{\prime}\right) \quad \forall\left(g ; g^{\prime}\right) \in G \times G \quad \forall f \in C^{\infty}(G, H, \beta) .
$$

We denote by $\mathcal{K}(G, H, \tau)$ the subspace of $C^{\infty}(G, H, \beta)$ of elements with compact support modulo $H$. Then the norm \|\|$_{\tau}$ on $\mathcal{K}(G, H, \tau)$ is given by

$$
\|f\|_{\tau}^{2}=\int_{H \backslash G}|f(g)|^{2} \mathrm{~d} \dot{\mathrm{g}}
$$

where dg denotes a right $G$-invariant measure on $H \backslash G$. The Hilbert space $\mathcal{H}_{\tau}$ is just the completion of $\mathcal{K}(G, H, \tau)$ relative to this norm. Moreover, the unitary

Received by the editors March 17, 2000.

2000 Mathematics Subject Classification. Primary 43A85, 22E27, 22E30.

(C)2001 American Mathematical Society 
representation of $G$ in $\mathcal{H}_{\tau}$ decomposes in a continuous sum of unitary irreducible representations of $G$,

$$
\tau \simeq \int_{\hat{G}}^{\oplus} m(\pi) \pi d \mu(\pi)
$$

where $m(\pi)$ denotes the multiplicity of $\pi$ and $d \mu$ a Plancherel measure on the unitary dual $\hat{G}$ of $G$. Then we know [3, 11] that for almost all $\pi$ in $\hat{G}$, the multiplicities $m(\pi)$ appearing in (1.4) either are finite and admit a uniform bound, or are all infinite. In the first case, we say that $\tau$ is of finite multiplicities.

Finally, let $\mathcal{D}(G, H, \beta)$ be the algebra of linear differential operators leaving $C^{\infty}(G, H, \beta)$ invariant and commuting with $\tau$, that is

$$
D \in \mathcal{D}(G, H, \beta) \Leftrightarrow D(\tau(g) f)=\tau(g)(D f) \quad \forall g \in G \quad \forall f \in C^{\infty}(G, H, \beta) .
$$

Corwin and Greenleaf established in [5] the commutativity of $\mathcal{D}(G, H, \beta)$ when $\tau$ is of finite multiplicities and they asked the question:

$$
\text { Is } \tau \text { of finite multiplicities if } \mathcal{D}(G, H, \beta) \text { is commutative? }
$$

Before we turn to the study of this question, we first recall some facts about parametrization of unipotent actions on vector spaces [12.

\section{Orbits OF $H$ IN $\mathfrak{g}^{*}$}

Suppose we are given a $m$-dimensional vector space $V$ admitting a unipotent action of $H$. Let $\left\{Y_{1} ; \cdots ; Y_{m}\right\}$ be a basis of $V$ such that the subspaces $V_{j} \equiv$ $\bigoplus_{i=j+1}^{m} \mathbb{R} Y_{i}$ of $V$ are $H$-stable for all $0 \leq j \leq m$ with $V_{m}=\{0\}$. We consider the multi-index $e(\psi)$ defined by $\left(e_{0}(\psi) ; \cdots ; e_{m}(\psi)\right)$ for all $\psi \in V$, where $e_{j}(\psi)$ is the dimension of the $H$-orbit of the projection of $\psi$ on $V / V_{j}$. We then denote by $\Sigma$ the set of all possible multi-indexes, that is

$$
\Sigma=\left\{e \in \mathbb{N}^{m+1} \mid \exists \psi \in V, \quad e=e(\psi)\right\} .
$$

This defines a stratification of $V$ in layers $U_{e}$ of $H$-orbits, more precisely 4 :

$$
V=\bigcup_{e \in \Sigma} U_{e} \text { where for } e \in \Sigma \text { and } U_{e}=\{\psi \in V \mid e(\psi)=e\} \text {. }
$$

It happens that among these layers $U_{e}, e \in \Sigma$, there exists one, and only one, which is a non-empty Zariski open subset of $V$. We shall call it the generic layer (associated to the action of $H$ on $V$ ), and we will denote it by $V^{g e n e}$. Note that $V^{g e n e}$ is just the subset of $V$ of elements for which the dimensions of $H$-orbits in $V / V_{j}$ are maximal for $0 \leq j \leq m$. We shall say that a $\psi$ in $V^{\text {gene }}$ is generic in $V$, and the dimension of the orbit $H \cdot \psi$ will be called the generic dimension of $H$-orbits in $V$.

In the sequel, we will consider a particular $V$. More precisely, fix a sequence $\{0\}=\mathfrak{g}_{0} \subset \mathfrak{g}_{1} \subset \cdots \subset \mathfrak{g}_{n}=\mathfrak{g}$ of subalgebras of $\mathfrak{g}$ satisfying the following conditions:

- the subalgebras $\mathfrak{g}_{i}$ are of dimension $i$; they are normalised by the action of $H$.

- for a certain index $p$, the subalgebra $\mathfrak{g}_{p}$ coincides with $\mathfrak{h}$.

We choose a weak Malcev basis $\left\{X_{i}, 1 \leq i \leq n\right\}$ of $\mathfrak{g}$ through $\mathfrak{h}$ associated to the sequence $\left(\mathfrak{g}_{i}, 0 \leq i \leq n\right)$ such that for all $i \in\{1, \ldots, n\}$, the vectors $\left\{X_{j}, 1 \leq j \leq i\right\}$ form a basis of $\mathfrak{g}_{i}$. We say that $\left\{X_{i}, 1 \leq i \leq n\right\}$ (resp. $\left(\mathfrak{g}_{i}, 0 \leq i \leq n\right)$ ) is a weak Malcev basis (resp. sequence of subalgebras) of $\mathfrak{g}$ through $\mathfrak{h}$. As usual we denote by $\left\{X_{i}^{*}, 1 \leq i \leq n\right\}$ the dual basis of $\left\{X_{i}, 1 \leq i \leq n\right\}$. Then we put $V=\mathfrak{g}^{*}$ and 
$V_{j}=\mathfrak{g}_{j}^{\perp}=\bigoplus_{k=j+1}^{n} \mathbb{R} X_{k}^{*}$ for $1 \leq j \leq n$. Here we consider the unipotent action of $H$ on $V=\mathfrak{g}^{*}$ given by the restriction to $H$ of the co-adjoint action of $G$ on $\mathfrak{g}^{*}$. In particular, the layer $V^{\text {gene }}$ defined before will be denoted by $\mathfrak{g}^{*, g e n e}$. This defines a stratification of $\mathfrak{g}^{*}$ in $U_{e}$-layers.

Next, denote by $\Omega_{G, H, \beta}$ the space of all continuations of $\beta$ to $V$. There is in $\left\{U_{e}, e \in \Sigma\right\}$ a unique layer intersecting $\Omega_{G, H, \beta}$ in a non-empty Zariski open subset (Section 2 of [5]). We will call it the generic layer associated to the data $G, H$ and $\beta$, and we will denote it by $\mathfrak{g}_{G, H, \beta}^{*, g e n e}$. We shall say that an element $\psi$ in $\Omega_{G, H, \beta}$ contained in $\mathfrak{g}_{G, H, \beta}^{*, g e n e}$ is generic in $\Omega_{G, H, \beta}$.

Remark 1. It is important to note that the layer $\mathfrak{g}^{*}$ gene does not necessarily intersect $\Omega_{G, H, \beta}$. Indeed, a family of simple examples where the condition $\mathfrak{g}^{* \text {,gene }} \cap$ $\Omega_{G, H, \beta} \neq \emptyset$ is not satisfied is the Heisenberg group with $\mathfrak{h}$ containing the center and $\beta$ vanishing on the center. More precisely, let $\mathfrak{g}=\mathbb{R} X \oplus \mathbb{R} Y \oplus \mathbb{R} Z$ with bracket relation $[X, Y]=Z$. If $\mathfrak{h}=\mathbb{R} X \oplus \mathbb{R} Z$ and $\beta=X^{*}$, then $\Omega_{G, H, \beta}=X^{*}+\mathbb{R} Y^{*}$ and $\mathfrak{g}^{* \text { gene }}=\left\{x X^{*}+y Y^{*}+z Z^{*} \in \mathfrak{g}^{*} \mid z \neq 0\right\}$, which shows that $\mathfrak{g}^{*, \text { gene }} \cap \Omega_{G, H, \beta}=\emptyset$.

Finally, for $l \in \mathfrak{g}^{*}$, we denote by $B_{l}$ the antisymmetric bilinear form on $\mathfrak{g}$ given by $B_{l}(X, Y)=l([X, Y])$. It is well known [3, 11] that the two following conditions are equivalent:

$(C 1) \tau$ is of finite multiplicities.

$(C 2) \mathfrak{h}+\mathfrak{g}(l)$ is lagrangian in $\mathfrak{g}$ relative to $B_{l}$ for all generic $l$ in $\Omega_{G, H, \beta}$.

If the second conditon is satisfied, we say that $\mathfrak{h}+\mathfrak{g}(l)$ is generically lagrangian in $\mathfrak{g}$. It turns out that question 6 asked by Duflo in [7] is, in the case of a simply connected connected real nilpotent Lie group, exactly the same as the above question $(\star)$ of Corwin-Greenleaf.

More precisely, consider the following assertions:

(i) $\mathcal{D}(G, H, \beta)$ is a commutative algebra.

(ii) $\mathfrak{h}+\mathfrak{g}(l)$ is generically lagrangian in $\mathfrak{g}$.

(iii) $H \cdot l$ is generically a lagrangian submanifold of $G \cdot l$ relative to $B_{l}:(X, Y) \mapsto$ $l([X, Y])$.

Note that $(i i) \Leftrightarrow(i i i)$ is obvious, since if $\mathfrak{h}+\mathfrak{g}(l)$ is lagrangian in $\mathfrak{g}$, then $\operatorname{dim} H \cdot l=$ $\frac{1}{2} \operatorname{dim} G \cdot l$. But $(i i) \Rightarrow(i)$ is a fundamental result proved by Corwin and Greenleaf in [5]. Baklouti and Ludwig have studied in [1] the implication $(i) \Rightarrow(i i)$ when $\mathfrak{h}$ is an ideal of $\mathfrak{g}$. In the sequel, we shall study the implication $(i) \Rightarrow(i i)$ in more general cases.

\section{A first Result on the COMmutativity of $\mathcal{D}(G, H, \beta)$}

The description of $\mathcal{D}(G, H, \beta)$ given in [6] in terms of the enveloping algebra $\mathcal{U}(\mathfrak{g})$ of the complexification of $\mathfrak{g}$ will be useful. Let $\mathfrak{a}_{\beta}$ be the vector subspace of $\mathcal{U}(\mathfrak{g})$ generated by the $X+2 \sqrt{-1} \pi \beta(X), X \in \mathfrak{h}$, and let $\mathcal{U}(\mathfrak{g}) \mathfrak{a}_{\beta}$ be the left sided ideal of $\mathcal{U}(\mathfrak{g})$ generated by $\mathfrak{a}_{\beta}$. If $\mathcal{U}(\mathfrak{g}, \mathfrak{h}, \beta)$ denotes the subalgebra of $\mathcal{U}(\mathfrak{g})$ defined by

$$
\mathcal{U}(\mathfrak{g}, \mathfrak{h}, \beta)=\left\{A \in \mathcal{U}(\mathfrak{g}) \mid \forall W \in \mathfrak{h}, \quad[A, W] \in \mathcal{U}(\mathfrak{g}) \mathfrak{a}_{\beta}\right\},
$$

then the left action $L$ of $\mathcal{U}(\mathfrak{g})$, defined for $Y$ in $\mathfrak{g}$ and $f$ in $C^{\infty}(G)$ by

$$
L(Y)(f)(g)=\left.\frac{d}{d t} f\left(e^{-t Y} g\right)\right|_{t=0},
$$

induces the algebra isomorphism $L_{\beta}: \mathcal{U}(\mathfrak{g}, \mathfrak{h}, \beta) / \mathcal{U}(\mathfrak{g}) \mathfrak{a}_{\beta} \simeq \mathcal{D}(G, H, \beta)$. 
Next, in the usual way, we shall denote by $S(\mathfrak{g})$ the symmetric algebra of $\mathfrak{g}$ and by $\sigma: S(\mathfrak{g}) \rightarrow \mathcal{U}(\mathfrak{g})$ the symmetrization map. We still denote by $A d$ (resp. ad) the natural continuation of the adjoint action of $G$ (resp. $\mathfrak{g}$ ) in a $G$-action (resp. $\mathfrak{g}$-action) on $S(\mathfrak{g})$ and $\mathcal{U}(\mathfrak{g})$. Moreover, we shall identify an element of $S(\mathfrak{g})$ with a polynomial function on $\mathfrak{g}^{*}$.

Remark 2. F. P. Greenleaf has also obtained the following theorem with a different proof [10].

Theorem 1. Let $G$ be a simply connected connected real nilpotent Lie group with Lie algebra $\mathfrak{g}, H$ a connected closed subgroup of $G$ with Lie algebra $\mathfrak{h}$ and $\beta \in \mathfrak{h}^{*}$ such that $\beta([\mathfrak{h}, \mathfrak{h}])=\{0\}$. We assume that the unitary representation $\tau=\operatorname{Ind}_{H}^{G} \chi_{\beta}$ of $G$ is of infinite multiplicities. Let $G_{0}$ be a connected subgroup of codimension one of $G$ with Lie algebra $\mathfrak{g}_{0}$ containing $\mathfrak{h}$ and such that the unitary representation $\tau_{0}=I n d_{H}^{G_{0}} \chi_{\beta}$ of $G_{0}$ is of finite multiplicities. If we suppose that there is an element $W$ of $\mathcal{U}(\mathfrak{g}, \mathfrak{h}, \beta)$ such that $W \notin \mathcal{U}\left(\mathfrak{g}_{0}\right)+\mathcal{U}(\mathfrak{g}) \mathfrak{a}_{\beta}$, then there exists an element $T$ of $\mathcal{U}\left(\mathfrak{g}_{0}, \mathfrak{h}, \beta\right)$ satisfying $[W, T] \notin \mathcal{U}(\mathfrak{g}) \mathfrak{a}_{\beta}$.

In other words, if $\mathcal{D}\left(G_{0}, H, \beta\right)$ is properly imbeded in $\mathcal{D}(G, H, \beta)$, then $\mathcal{D}(G, H, \beta)$ is not commutative.

Proof. We shall frequently use in the sequel the following property: let $\mathfrak{k}$ be a subalgebra of codimension one of $\mathfrak{g}$, and $l$ be in $\mathfrak{g}^{*}$, then the dimensions of $\mathfrak{g}(l)$ and of $\mathfrak{k}\left(l_{l_{\mathfrak{k}}}\right)$ differ by 1 , and one of those subspaces is imbedded in the other, (see [2], Lemme 1.1.1, p. 49). In the case where $\mathfrak{g}(l) \subset \mathfrak{k}\left(l_{\mathfrak{k}}\right)$, one has that the dimension of $G . l$ is bigger by 2 than the dimension of $K .\left(l_{l_{\mathfrak{k}}}\right)$ where $K=\exp (\mathfrak{k})$, and the dimensions of polarizations in $\mathfrak{g}$ and $\mathfrak{k}$ are the same. In the other case, the orbits $G . l$ and $K .\left(l_{\left.\right|_{\mathfrak{k}}}\right)$ have the same dimension, whereas the dimension of polarizations in $\mathfrak{g}$ is bigger by 1 than the dimension of polarizations in $\mathfrak{k}$.

Let us recall that the finite multiplicities situation is characterised by the fact that generically on $\Omega_{G, H, \beta}$, the dimension of an $H$-orbit is half the dimension of a $G$-orbit [5] . Thus, under the assumptions of the theorem, we have $\mathfrak{g}(l) \subset \mathfrak{g}_{0}$.

Next, we proceed by induction on the dimension of $G$ and the theorem is supposed to be true for all groups of dimension at most $n-1$, where $n$ is the dimension of $G$. Let $\mathcal{Z}$ be the center of $\mathfrak{g}$. We shall consider two main cases depending on whether $\mathcal{Z}$ is included in $\mathfrak{h}$.

1) Case: $\mathcal{Z} \subset \mathfrak{h}$ and $\mathcal{Z} \cap \operatorname{Ker}(\beta) \neq\{0\}$. In this case, we apply the induction hypothesis to the quotient group with Lie algebra $\mathfrak{g} /(\mathcal{Z} \cap \operatorname{Ker}(\beta))$.

In the following cases 2),3) and 4), we have $\mathcal{Z} \subset \mathfrak{h}$ and $\mathcal{Z} \cap \operatorname{Ker}(\beta)=\{0\}$, so that the center $\mathcal{Z}$ of $\mathfrak{g}$ is necessarily one-dimensional. We put $\mathcal{Z}=\mathbb{R} Z$ with $\beta(Z)=1$. Moreover, it is easy to check the existence of elements $X$ of $\mathfrak{g}$ and $Y$ of $\mathfrak{g}_{0}$ such that $[X, Y]=Z$. In the sequel, we shall denote by $\mathfrak{k}$ the centralizer of $Y$ in $\mathfrak{g}$ and by $K$ the connected subgroup of $G$ with Lie algebra $\mathfrak{k}$.

2) Case: $\mathcal{Z} \subset \mathfrak{h}, \mathcal{Z} \cap \operatorname{Ker}(\beta)=\{0\}, \mathfrak{h} \subset \mathfrak{k}$ and $Y \in \mathfrak{h}$. Let $l$ be an element of $\mathfrak{g}^{*}$ satisfying $l(Z) \neq 0$, (since $\beta(Z)=1$, this condition is satisfied by any element of $\left.\Omega_{G, H, \beta}\right)$. One has $\mathfrak{k}\left(l_{\mid \mathfrak{k}}\right)=\mathfrak{g}(l) \oplus \mathbb{R} Y$. And, as we noticed before, the dimension $[\operatorname{dim}(\mathfrak{g})+\operatorname{dim}(\mathfrak{g}(l))] / 2$ of a polarization of $\mathfrak{g}$ at a point $l \in \mathfrak{g}^{*}$ is the same as the dimension $\left[\operatorname{dim}(\mathfrak{k})+\operatorname{dim}\left(\mathfrak{k}\left(l_{\left.\right|_{\mathfrak{k}}}\right)\right)\right] / 2$ of a polarization of $\mathfrak{k}$ at the point $l_{l_{\mathfrak{k}}} \in \mathfrak{k}^{*}$. Moreover, we also have $\mathfrak{h}+\mathfrak{k}\left(l_{\mid \mathfrak{k}}\right)=\mathfrak{h}+\mathfrak{g}(l)$. Next, we choose a weak Malcev basis of 
$\mathfrak{g}$ passing throught $\mathfrak{h}$ and $\mathfrak{k}$, and we consider a generic element $l$ in $\Omega_{G, H, \beta}$. Then, the equivalent conditions $(C 1)$ and $(C 2)$ of Section 2 show that the multiplicities of the representations $\tau=\operatorname{Ind} d_{H}^{G} \chi_{\beta}$ of $G$ and $\tau^{\prime}=\operatorname{Ind} d_{H}^{K} \chi_{\beta}$ of $K$ are of the same type, that is, both infinite. But $\tau_{0}=\operatorname{Ind} d_{H}^{G_{0}} \chi_{\beta}$ is supposed to be of finite multiplicities, so one has $\mathfrak{g}_{0} \neq \mathfrak{k}$.

On the other hand, for $l \in \Omega_{G, H, \beta}$, we have $\left(\mathfrak{g}_{0} \cap \mathfrak{k}\right)\left(l_{\left.\right|_{\mathfrak{g}_{0} \cap \mathfrak{k}}}\right)=\mathfrak{g}_{0}\left(l_{\left.\right|_{\mathfrak{g}_{0}}}\right) \oplus \mathbb{R} Y$. Choosing a weak Malcev basis of $\mathfrak{g}$ passing through $\mathfrak{h}, \mathfrak{g}_{0} \cap \mathfrak{k}$ and $\mathfrak{g}_{0}$, we see that the unitary representation $\tau_{0}^{\prime}=\operatorname{Ind} d_{H}^{G_{0} \cap K} \chi_{\beta}$ of $G_{0} \cap K$ is, as the representation $\tau_{0}=\operatorname{Ind} d_{H}^{G_{0}} \chi_{\beta}$ of $G_{0}$, of finite multiplicities. Let us write the element $W$ of the theorem in the form $W=\sum_{j=0}^{j=r} X^{j} U_{j}$ with $U_{j}$ in $\mathcal{U}(\mathfrak{k})$. Since the element $Y$ is in $\mathfrak{h}$, the elements $a d(-Y)^{r}(W)=r ! Z^{r} U_{r}$ and $U_{r}$ are in $\mathcal{U}(\mathfrak{g}) \mathfrak{a}_{\beta}$ for all $r \neq 0$. Thus we can suppose $W=U_{0}$ is in $\mathcal{U}(\mathfrak{k}, \mathfrak{h}, \beta)$. Finally, for $W \in \mathcal{U}(\mathfrak{k}, \mathfrak{h}, \beta)$, we apply the induction hypothesis to $K$ and $G_{0} \cap K$ with the representation $\tau^{\prime}=\operatorname{Ind}_{H}^{K} \chi_{\beta}$ and $\tau_{0}^{\prime}=\operatorname{Ind} d_{H}^{G_{0} \cap K} \chi_{\beta}$ respectively.

3) Case: $\mathcal{Z} \subset \mathfrak{h}, \mathcal{Z} \cap \operatorname{Ker}(\beta)=\{0\}, \mathfrak{h} \subset \mathfrak{k}$ and $Y \notin \mathfrak{h} . i) \mathfrak{g}_{0}=\mathfrak{k}$.

The element $Y$ is in $\mathcal{U}\left(\mathfrak{g}_{0}, \mathfrak{h}, \beta\right)$ and satisfy $[W, Y] \notin \mathcal{U}(\mathfrak{g}) \mathfrak{a}_{\beta}$. We take $T=Y$.

ii) $\mathfrak{g}_{0} \neq \mathfrak{k}$ and $W \notin \mathcal{U}(\mathfrak{k})+\mathcal{U}(\mathfrak{g}) \mathfrak{a}_{\beta}$.

We still can choose $T$ to be the element $Y$.

iii) $\mathfrak{g}_{0} \neq \mathfrak{k}$ and $W \in \mathcal{U}(\mathfrak{k})+\mathcal{U}(\mathfrak{g}) \mathfrak{a}_{\beta}$.

We consider the inclusions: $\left(\mathfrak{g}_{0} \cap \mathfrak{k}\right) \subset \mathfrak{g}_{0} \subset \mathfrak{g}$ and $\left(\mathfrak{g}_{0} \cap \mathfrak{k}\right) \subset \mathfrak{k} \subset \mathfrak{g}$. Let $l$ be in $\mathfrak{g}^{*}$ with $l(Z) \neq 0$. We denote by $d$ the dimension of $\mathfrak{g}(l)$. Under the assumption of the theorem, as we have already remarked at the beginning, we have $\mathfrak{g}(l) \subset \mathfrak{g}_{0}$, so that the dimension of $\mathfrak{g}_{0}\left(l_{\left.\right|_{\mathfrak{g}_{0}}}\right)$ is $d+1$. The dimension of $\mathfrak{k}\left(l_{\left.\right|_{\mathfrak{k}}}\right)$ is also $d+1$ because the element $Y$ of $\mathfrak{k}\left(l_{\left.\right|_{\mathfrak{k}}}\right)$ does not belong to $\mathfrak{g}(l)$. Moreover, $Y$ is in $\left(\mathfrak{g}_{0} \cap \mathfrak{k}\right)\left(\left.l\right|_{\left(\mathfrak{g}_{0} \cap \mathfrak{k}\right)}\right)$ but is not in $\mathfrak{g}_{0}\left(l_{\left.\right|_{\mathfrak{g}_{0}}}\right)$. In other words, the dimension of $\left(\mathfrak{g}_{0} \cap \mathfrak{k}\right)\left(\left.l\right|_{\left(\mathfrak{g}_{0} \cap \mathfrak{k}\right)}\right)$ is $d+2$. Thus, the representation $\tau^{\prime}=\operatorname{In} d_{H}^{K} \chi_{\beta}$ of $K$ is necessarily of infinite multiplicities. Indeed, the representation $\tau_{0}^{\prime}=\operatorname{Ind}_{H}^{K \cap G_{0}} \chi_{\beta}$ has finite multiplicities and, from the previous calculus of dimensions of stabilizers, we deduce that if the representation $\tau^{\prime}=I n d_{H}^{K} \chi_{\beta}$ of $K$ had finite multiplicities, the dimensions of $H$-orbits for generic $l \in \Omega_{G, H, \beta}$ would increase when passing from $K \cap G_{0}$ to $K$, which makes impossible the existence of an element $W \in \mathcal{U}(\mathfrak{k})+\mathcal{U}(\mathfrak{g}) \mathfrak{a}_{\beta}$ that is not in $\mathcal{U}\left(\mathfrak{k} \cap \mathfrak{g}_{0}\right)+\mathcal{U}(\mathfrak{g}) \mathfrak{a}_{\beta}$ as it is shown in [5]. Finally, we apply the induction hypothesis to $K$ and $G_{0} \cap K$ with the representation $\tau^{\prime}$ and $\tau_{0}^{\prime}$ respectively.

4) Case: $\mathcal{Z} \subset \mathfrak{h}, \mathcal{Z} \cap \operatorname{Ker}(\beta)=\{0\}$ and $\mathfrak{h} \not \subset \mathfrak{k}$. Note that, since $\mathfrak{h} \subset \mathfrak{g}_{0}$, one has necessarily $\mathfrak{g}_{0} \neq \mathfrak{k}$. Let us write $\mathfrak{g}_{0}=\left(\mathfrak{g}_{0} \cap \mathfrak{k}\right) \oplus \mathbb{R} X$, with $X \in \mathfrak{h}$, so that $\mathfrak{g}=\mathfrak{k} \oplus \mathbb{R} X$, and denote by $\hat{\beta}$ the restriction of $\beta$ to $\mathfrak{h} \cap \mathfrak{k}$. Let $l$ be an element of $\Omega_{G, H, \beta}$. We have $l(Z) \neq 0$. Then $\mathfrak{k}\left(l_{\left.\right|_{\mathfrak{k}}}\right)=\mathfrak{g}(l) \oplus \mathbb{R} Y$, so that $(\mathfrak{h} \cap \mathfrak{k}) \cap \mathfrak{k}\left(l_{\left.\right|_{\mathfrak{k}}}\right)=\mathfrak{h} \cap \mathfrak{g}(l)$, since $\mathfrak{h} \not \subset \mathfrak{k}$. This forces the vector spaces $\mathfrak{h}+\mathfrak{g}(l)$ and $(\mathfrak{h} \cap \mathfrak{k})+\mathfrak{k}\left(l_{\left.\right|_{\mathfrak{k}}}\right)$ to have the same dimension. It follows that if the first subspace is not lagrangian in $\mathfrak{g}$, the second is not lagrangian in $\mathfrak{k}$. Hence, choosing sequences of subalgebras and using equivalence of conditions $(C 1)$ and $(C 2)$, we obtain that the representation $\tau_{1}=\operatorname{Ind} d_{H \cap K}^{K} \chi_{\hat{\beta}}$ of $K$ is of infinite multiplicities. On the other hand, we have $\left(\mathfrak{g}_{0} \cap \mathfrak{k}\right)\left(\left.l\right|_{\mathfrak{g}_{0} \cap \mathfrak{k}}\right)=\mathfrak{g}_{0}\left(l_{\left.\right|_{\mathfrak{g}_{0}}}\right) \oplus \mathbb{R} Y$ and $\operatorname{dim}\left(\mathfrak{h}+\mathfrak{g}_{0}\left(\left.l\right|_{\mathfrak{g}_{0}}\right)\right)=\operatorname{dim}\left((\mathfrak{h} \cap \mathfrak{k})+\left(\mathfrak{g}_{0} \cap \mathfrak{k}\right)\left(\left.l\right|_{\mathfrak{g}_{0} \cap \mathfrak{k}}\right)\right)$, which imply that the representation $\tau_{2}=I n d_{H \cap K}^{G_{0} \cap K} \chi_{\hat{\beta}}$ of $G_{0} \cap K$ is of finite multiplicities.

Moreover, $W$ can be supposed to belong to $\mathcal{U}(\mathfrak{k}, \mathfrak{h} \cap \mathfrak{k}, \hat{\beta})$ since $\mathfrak{g}=\mathfrak{k} \oplus \mathbb{R} X$ with $X \in \mathfrak{h}$, and $X=-2 \sqrt{-1} \pi \beta(X)$ modulo $\mathfrak{a}_{\beta}$. We apply the induction hypothesis to 
$K$ and $G_{0} \cap K$ with the representation $\tau_{1}$ and $\tau_{2}$ respectively to obtain an element $\hat{T}$ in $\mathcal{U}\left(\mathfrak{g}_{0} \cap \mathfrak{k}, \mathfrak{h} \cap \mathfrak{k}, \hat{\beta}\right)$ such that $[W, \hat{T}] \notin \mathcal{U}(\mathfrak{k}) \mathfrak{a}_{\hat{\beta}}$.

Next, let $X_{1}=Y$ and $X_{i}, i \in\{2, \cdots, q\}$, be in $\mathfrak{g}_{0} \cap \mathfrak{k}$, such that if we put $\tilde{\mathfrak{g}_{i}}=\mathfrak{h} \oplus \mathbb{R} X_{1} \oplus \mathbb{R} X_{2} \oplus \cdots \oplus \mathbb{R} X_{i}$ with $\mathfrak{g}_{q}=\mathfrak{g}_{0}$, then the sequence of subalgebras $\left(\tilde{\mathfrak{g}}_{i}\right)_{i=1, \cdots, q}$ of $\mathfrak{g}_{0}$ is Jordan-Hölder for the action of $H$ on $\mathfrak{g}_{0}$. It is interesting to notice that the sequence $\left(\hat{\mathfrak{g}}_{i}\right)_{i=1, \cdots, q}$, with $\hat{\mathfrak{g}}_{i}=(\mathfrak{h} \cap \mathfrak{k}) \oplus \mathbb{R} X_{1} \oplus \cdots \oplus \mathbb{R} X_{i}$ is also Jordan-Hölder for the action of $H \cap K$ on $\mathfrak{g}_{0} \cap \mathfrak{k}$. We put $\tilde{G}_{i}=\exp \left(\tilde{\mathfrak{g}}_{i}\right)$ and $\hat{G}_{i}=\exp \left(\hat{\mathfrak{g}}_{i}\right)$ for $i=1, \cdots, q$. Then the dimension of generic $H$-orbits in $\Omega_{\tilde{G}_{j}, H, \beta}$ is bigger by one than that of generic $H \cap K$-orbits in $\Omega_{\hat{G}_{j}, H \cap K, \hat{\beta}}$.

On the other hand, since the representations $\tau_{0}=\operatorname{Ind}_{H}^{G_{0}} \chi_{\beta}$ of $G_{0}$ and $\tau_{2}=$ $\operatorname{Ind} d_{H \cap K}^{G_{0} \cap K} \chi_{\hat{\beta}}$ of $G_{0} \cap K$ are of finite multiplicities, there are elements $\left\{\tilde{\gamma}_{1}, \cdots, \tilde{\gamma}_{r}\right\}$ of $\mathcal{U}\left(\mathfrak{g}_{0}, \mathfrak{h}, \beta\right)$ and $\left\{\hat{\delta_{0}}=Y, \hat{\delta_{1}}, \cdots, \hat{\delta_{r}}\right\}$ of $\mathcal{U}\left(\mathfrak{g}_{0} \cap \mathfrak{k}, \mathfrak{h} \cap \mathfrak{k}, \hat{\beta}\right)$ given in [5], such that the families $\left\{\gamma_{i}=L\left(\tilde{\gamma}_{i}\right) \mid i=1, \cdots, r\right\}$ and $\left\{\delta_{i}=L\left(\hat{\delta}_{i}\right) \mid i=0, \cdots, r\right\}$ are rational generators of $\mathcal{D}\left(G_{0}, H, \beta\right)$ and $\mathcal{D}\left(G_{0} \cap K, H \cap K, \hat{\beta}\right)$ respectively. To simplify, we shall call the $\gamma_{i}$ 's and $\delta_{i}$ 's, the Corwin-Greenleaf generators of $\mathcal{D}\left(G_{0}, H, \beta\right)$ and $\mathcal{D}\left(G_{0} \cap K, H \cap K, \hat{\beta}\right)$ respectively. Note that $\mathcal{D}\left(G_{0}, H, \beta\right)$ is contained in $\mathcal{D}\left(G_{0} \cap K, H \cap K, \hat{\beta}\right)$. Moreover, we may suppose that for the element $\hat{T}$ above, $L(\hat{T})$ is one of the Corwin-Greenleaf generators of $\mathcal{D}\left(G_{0} \cap K, H \cap K, \hat{\beta}\right)$. Thus, since $[W, Y]=0$, we denote by $\delta_{j_{0}}$ the first element of $\left\{\hat{\delta_{1}}, \cdots, \hat{\delta_{r}}\right\}$ satisfying $\left[\hat{\delta}_{j_{0}}, W\right] \notin \mathcal{U}(\mathfrak{k}) \mathfrak{a}_{\hat{\beta}}$. Then, from [5], one can find polynomials $A, B$ and $C$ of $j_{0}$ variables such that $A\left(\delta_{0}, \cdots, \delta_{j_{0}-1}\right) \gamma_{j_{0}}=B\left(\delta_{0}, \cdots, \delta_{j_{0}-1}\right) \delta_{j_{0}}+C\left(\delta_{0}, \cdots, \delta_{j_{0}-1}\right)$, with $A\left(\delta_{0}, \cdots, \delta_{j_{0}-1}\right)$ and $B\left(\delta_{0}, \cdots, \delta_{j_{0}-1}\right)$ non-zero.

It turns out that $\left[\tilde{\gamma}_{j_{0}}, W\right] \notin \mathcal{U}(\mathfrak{g}) \mathfrak{a}_{\beta}$. Indeed, because $\mathcal{D}(G, H, \beta)$ has no non-zero divisor of zero, we have $A\left(\delta_{1}, \cdots, \delta_{j_{0}-1}\right)\left[\gamma_{j_{0}}, L(W)\right]=B\left(\delta_{1}, \cdots, \delta_{j_{0}-1}\right)\left[\delta_{j_{0}}, L(W)\right]$ $\neq 0$, so that $\left[\gamma_{j_{0}}, L(W)\right] \neq 0$. Hence, we can choose $T=\tilde{\gamma}_{j_{0}}$, that is $L(T)$ is the Corwin-Greenleaf generator $\gamma_{j_{0}}$ of $\mathcal{D}\left(G_{0}, H, \beta\right)$.

5) Case: $\mathcal{Z} \not \subset \mathfrak{h}$. First, remember that an immediate consequence of the assumptions of the theorem is that $\mathcal{Z}$ is embeded in $\mathfrak{g}_{0}$. Next, let $Z$ be in $\mathcal{Z}$ which does not belong to $\mathfrak{h}$. Denote by $\mathfrak{h}^{\prime}$ the subalgebra $\mathfrak{h} \oplus \mathbb{R} Z$ of $\mathfrak{g}$ and by $H^{\prime}$ the connected subgroup of $G$ with Lie algebra $\mathfrak{h}^{\prime}$. Let $\phi$ be a generic element of $\Omega_{G, H, \beta}$ and put $\alpha=\phi(Z)$. Define, as usual, the character $\chi_{\phi}$ of $H^{\prime}$ by $\chi_{\phi}\left(e^{U}\right)=e^{2 \sqrt{-1} \pi \phi(U)}$ for all $U \in \mathfrak{h}^{\prime}$, so that the unitary representation $\tau_{0}^{\alpha}=\operatorname{Ind}_{H^{\prime}}^{G_{0}} \chi_{\phi}$ of $G_{0}$ is of finite multiplicities. Let $\mathfrak{h} \subset \mathfrak{h} \oplus \mathbb{R} Z \subset \mathfrak{h} \oplus \mathbb{R} Z \oplus \mathbb{R} X_{1} \subset \mathfrak{h} \oplus \mathbb{R} Z \oplus \mathbb{R} X_{1} \oplus \mathbb{R} X_{2} \subset \cdots \subset \mathfrak{g}_{0}$ be a Jordan-Hölder sequence for the action of $H$ on $\mathfrak{g}_{0}$, and consider the sequence $\mathfrak{h}^{\prime} \subset \mathfrak{h}^{\prime} \oplus \mathbb{R} X_{1} \subset \mathfrak{h}^{\prime} \oplus \mathbb{R} X_{1} \oplus \mathbb{R} X_{2} \subset \cdots \subset \mathfrak{g}_{0}$ which is also a JordanHölder sequence for the action of $H^{\prime}$ on $\mathfrak{g}_{0}$. Actually, since $H$ and $H^{\prime}$ have the same orbits in $\mathfrak{g}_{0}$, if $\left\{\gamma_{1}=L(Z), \gamma_{2}, \cdots, \gamma_{q}\right\}$ is a set of Corwin-Greenleaf generators of $D\left(G_{0}, H, \beta\right)$, then $\left\{\gamma_{2}, \cdots, \gamma_{q}\right\}$ is a set of Corwin-Greenleaf generators of $D\left(G_{0}, H^{\prime}, \phi\right)$. Any Corwin-Greenleaf generator of $D\left(G_{0}, H, \beta\right)$ can be represented in $\mathcal{U}\left(\mathfrak{g}_{0}, \mathfrak{h}, \beta\right) / \mathcal{U}\left(\mathfrak{g}_{0}\right) \mathfrak{a}_{\beta}$ by an element $C=\sum_{\nu, \mu} a_{\nu, \mu} Z^{\nu} X_{1}^{\mu_{1}} \cdots X_{p}^{\mu_{p}}$ of $\mathcal{U}\left(\mathfrak{g}_{0}, \mathfrak{h}, \beta\right)$. And observe that any element of $\mathcal{U}\left(\mathfrak{g}_{0}, \mathfrak{h}, \beta\right)$ belongs to $\mathcal{U}\left(\mathfrak{g}_{0}, \mathfrak{h}^{\prime}, \phi\right)$. Actually, $C$ acts on $C^{\infty}\left(G_{0}, H^{\prime}, \phi\right)$ as $C(\alpha)=\sum_{\nu, \mu} a_{\nu, \mu}(-2 \sqrt{-1} \pi \alpha)^{\nu} X_{1}^{\mu_{1}} \cdots X_{p}^{\mu_{p}}$. On the other hand, the element $W$ of $\mathcal{U}(\mathfrak{g}, \mathfrak{h}, \beta)$ acts on $C^{\infty}\left(G, H^{\prime}, \phi\right)$ as $W(\alpha)$, so that $[W(\alpha), C(\alpha)]=[W, C](\alpha)$ on $C^{\infty}\left(G, H^{\prime}, \phi\right)$. Moreover, one can choose $\alpha$ in such a way that $W=W(\alpha)+(W-W(\alpha))=W(\alpha)+\tilde{W}[Z+2 \sqrt{-1} \pi \alpha]$, with $\tilde{W} \in \mathcal{U}(\mathfrak{g})$ 
and $W(\alpha) \notin \mathcal{U}\left(\mathfrak{g}_{0}\right)+\mathcal{U}(\mathfrak{g}) \mathfrak{a}_{\phi}$. If $\mathcal{Z} \not \subset \mathfrak{h}^{\prime}$, taking an element in $\mathcal{Z}$ which does not belong to $\mathfrak{h}^{\prime}$, we apply the same procedure as above. After a finite number of steps, we get, instead of $\mathfrak{h}$, a subalgebra containing the center $\mathcal{Z}$ of $\mathfrak{g}$. In this case, we just apply the results of the previous cases and we choose for the element $T$ one of the Corwin-Greenleaf generators.

3.1. The case where $(\mathfrak{g} ; \mathfrak{h})$ is a reductive pair. We say that $(\mathfrak{g} ; \mathfrak{h})$ is a reductive pair, if there exists a vector subspace $\mathfrak{m}$ of $\mathfrak{g}$ such that $\mathfrak{g}=\mathfrak{h} \oplus \mathfrak{m}$ and $[\mathfrak{h}, \mathfrak{m}] \subset \mathfrak{m}$.

Corollary 1. Let $G$ be a simply connected connected real nilpotent Lie group with Lie algebra $\mathfrak{g}, H$ a connected closed subgroup of $G$ with Lie algebra $\mathfrak{h}$ and $\beta \in \mathfrak{h}^{*}$ such that $\beta([\mathfrak{h}, \mathfrak{h}])=\{0\}$. Suppose that $(\mathfrak{g} ; \mathfrak{h})$ is a reductive pair. Then the assertions (i), (ii) and (iii) of Section 2 are equivalent.

Proof. $(i) \Rightarrow(i i)$ : Under the notation of Section 2, we take $V=\mathfrak{m}^{*}$, which could be identified to $\Omega_{G, H, \beta}$, so that we have the unipotent action $A d^{*}$ of $H$ on $V$. Let $\mathfrak{g}_{0}$ be an ideal of codimension one of $\mathfrak{g}$ containing $\mathfrak{h}$ and $G_{0}=\exp \left(\mathfrak{g}_{0}\right)$. One can suppose that $\tau_{0}=\operatorname{Ind} d_{H}^{G_{0}} \chi_{\beta}$ is of finite multiplicities. Then, the $H$-orbits in $V$ have generically the same dimension as the $H$-orbits in $\left(\mathfrak{m} \cap \mathfrak{g}_{0}\right)^{*}$ (that is the $H$ orbits in $V$ are generically not saturated in the direction $\left.\left(\mathfrak{m} \cap \mathfrak{g}_{0}\right)^{\perp}\right)$. This implies the existence of an $H$-invariant homogeneous polynomial $\mathcal{P}$ on $V$ which does not belong to $S\left(\mathfrak{m} \cap \mathfrak{g}_{0}\right)$ (Theorem of page 55 in [12]). On the other hand, since $(\mathfrak{g} ; \mathfrak{h})$ is a reductive pair then it is easy to check that the symmetrization map $\sigma$ is a vector space isomorphism between $S(\mathfrak{m})^{H}$ and $\mathcal{U}(\mathfrak{g}, \mathfrak{h}, \beta) / \mathcal{U}(\mathfrak{g}) \mathfrak{a}_{\beta}$. In particular, $\sigma(\mathcal{P})$ is a non-zero element of $\mathcal{U}(\mathfrak{g}, \mathfrak{h}, \beta)$ satisfying $\sigma(\mathcal{P}) \notin \mathcal{U}\left(\mathfrak{g}_{0}\right)+\mathcal{U}(\mathfrak{g}) \mathfrak{a}_{\beta}$. So Theorem 1 above applies to $W \equiv \sigma(\mathcal{P})$.

For $(i i) \Rightarrow(i i i)$ and $(i i i) \Rightarrow(i)$ see the end of Section 2 .

Remark 3. An interesting consequence of the Corollary 1 is that if $H$ is onedimensional then the assertions $(i),(i i)$ and $(i i i)$ are equivalent. Indeed, if $\mathfrak{h}$ is a one-dimensional subalgebra of $\mathfrak{g}$, then it is easy to see that $(\mathfrak{g} ; \mathfrak{h})$ is a reductive pair [5].

Remark 4. Another consequence of the Corollary 1 is the case where $\operatorname{Ker}(\beta)$ is an ideal of $\mathfrak{g}$. In this case, we just apply the Remark 3 above to the one-dimensional quotient $\mathfrak{h} / \operatorname{Ker}(\beta)$.

\section{A second Result on the Commutativity of $\mathcal{D}(G, H, \beta)$}

Here we explain, precisely, how to construct, in some cases, the element $W$ of Theorem 1. We keep the previous notation. In particular, remember that $\sigma$ : $S(\mathfrak{g}) \rightarrow \mathcal{U}(\mathfrak{g})$ denotes the symmetrization map, and $L$ is the left action of $\mathcal{U}(\mathfrak{g})$ on $C^{\infty}(G)$ defined by $(3.2)$.

\subsection{Preliminary results.}

Lemma 1. Let $\mathfrak{m}$ be an ideal of codimension one in $\mathfrak{g}$ such that $\mathfrak{g}=\mathfrak{m} \oplus \mathbb{R} X$. If $P \in S(\mathfrak{m})$, then $\sigma(P X)=\sigma(P) X+Q$ where $Q \in \mathcal{U}(\mathfrak{m})$.

Proof. Let $k \geq 1$. If $I_{k}=\left(i_{1}, \cdots, i_{k}\right) \in[1 ; m]^{k}$, we define $P_{I_{k}}=X_{i_{1}} \cdots X_{i_{k}}$. Let $P \in S(\mathfrak{m})$ be of degree $d$, such that $P=\sum_{k=1}^{d} \sum_{I_{k} \in[1 ; m]^{k}} a_{I_{k}} P_{I_{k}}$, with $a_{I_{k}} \in \mathbb{C}$. 
Thus, we have

$$
\sigma(P X)=\sum_{k=1}^{d} \sum_{I_{k} \in[1 ; m]^{k}} a_{I_{k}} \frac{1}{(k+1) !} \sum_{\mu \in \mathcal{S}_{k}} \sum_{j=0}^{k} T_{j}\left(X_{\mu\left(i_{1}\right)} \cdots X_{\mu\left(i_{k}\right)}\right)
$$

where $\mathcal{S}_{k}$ denotes the symmetric group of $k$ elements, and, for all $0 \leq j \leq k$, $T_{j}\left(X_{\mu\left(i_{1}\right)} \cdots X_{\mu\left(i_{k}\right)}\right) \equiv X_{\mu\left(i_{1}\right)} \cdots X_{\mu\left(i_{j}\right)} X X_{\mu\left(i_{j+1}\right)} \cdots X_{\mu\left(i_{k}\right)}$. Remarking that

$$
T_{j}\left(X_{\mu\left(i_{1}\right)} \cdots X_{\mu\left(i_{k}\right)}\right)=T_{j+1}\left(X_{\mu\left(i_{1}\right)} \cdots X_{\mu\left(i_{k}\right)}\right)+q, q \in \mathcal{U}(\mathfrak{m}),
$$

we get that

$$
T_{j}\left(X_{\mu\left(i_{1}\right)} \cdots X_{\mu\left(i_{k}\right)}\right)=X_{\mu\left(i_{1}\right)} \cdots X_{\mu\left(i_{k}\right)} X+\tilde{q}, \quad \tilde{q} \in \mathcal{U}(\mathfrak{m}),
$$

so

$$
\begin{aligned}
\sigma(P X) & =\sum_{k=1}^{d} \sum_{I_{k} \in[1 ; m]^{k}} a_{I_{k}} \frac{1}{k !} \sum_{\mu \in \mathcal{S}_{k}} X_{\mu\left(i_{1}\right)} \cdots X_{\mu\left(i_{k}\right)} X+Q \\
& =\sigma(P) X+Q, \quad Q \in \mathcal{U}(\mathfrak{m}) .
\end{aligned}
$$

Now let $\left\{X_{1}, \cdots, X_{n}\right\}$ be a basis of $\mathfrak{g}$, such that $\left\{X_{1}, \cdots, X_{p}\right\}$ is a basis of $\mathfrak{h}$ and

$$
\left[X_{j}, X_{k}\right]=\sum_{l=1}^{\sup (j, k)-1} a_{l}(j, k) X_{l} \quad \text { with } a_{l}(j, k) \in \mathbb{R} .
$$

Moreover, for all $f$ in $C^{\infty}(G, H, \beta)$, we define a function $f^{\sharp}$ on $\mathbb{R}^{n}$ by

$$
f^{\sharp}\left(x_{1}, \cdots, x_{n}\right)=f\left(\exp \left(x_{1} X_{1}\right) \cdots \exp \left(x_{n} X_{n}\right)\right) .
$$

If $X_{j}$ is in $\mathfrak{g}$, we put

$$
\left[L\left(X_{j}\right)\right]^{\sharp}\left(f^{\sharp}\right)=\left[L\left(X_{j}\right)(f)\right]^{\sharp} \quad \forall f \in C^{\infty}(G, H, \beta) .
$$

This definition extends naturally to $\mathcal{U}(\mathfrak{g})$.

Lemma 2. We have

$\left[L\left(X_{j}\right)\right]^{\sharp}=\left\{\begin{array}{l}-2 \sqrt{-1} \pi \beta\left(X_{j}\right) I d \quad \text { if } 1 \leq j \leq p, \\ -\frac{\partial}{\partial x_{j}}-\sum_{l=p+1}^{j-1} q_{l} \frac{\partial}{\partial x_{l}}-2 \sqrt{-1} \pi \sum_{l=1}^{p} q_{l} \beta\left(X_{l}\right) \text { Id if } p+1 \leq j \leq n,\end{array}\right.$

where the $q_{l}$ are polynomials in variables $x_{1}, \cdots, x_{j-1}$ such that $q_{l}(0)=0$.

Proof. Let $f \in C^{\infty}(G, H, \beta)$. By definition, we have

$$
\begin{aligned}
& {\left[L\left(X_{j}\right)\right] f\left(\exp \left(x_{1} X_{1}\right) \cdots \exp \left(x_{n} X_{n}\right)\right)} \\
& \quad=\left.\frac{d}{d t} f\left(\exp \left(-t X_{j}\right) \exp \left(x_{1} X_{1}\right) \cdots \exp \left(x_{n} X_{n}\right)\right)\right|_{t=0} .
\end{aligned}
$$

Then we have to consider the two cases $1 \leq j \leq p$ and $p+1 \leq j \leq n$.

Case: $1 \leq j \leq p$. Since $f$ is $H$-covariant, it follows that

$$
\begin{aligned}
& {\left[L\left(X_{j}\right)\right] f\left(\exp \left(x_{1} X_{1}\right) \cdots \exp \left(x_{n} X_{n}\right)\right)} \\
& \quad=\left.\frac{d}{d t} \exp \left(-2 \sqrt{-1} \pi t \beta\left(X_{j}\right)\right) f\left(\exp \left(x_{1} X_{1}\right) \cdots \exp \left(x_{n} X_{n}\right)\right)\right|_{t=0}
\end{aligned}
$$


which means that

$$
\left[L\left(X_{j}\right)\right]^{\sharp}=-2 \sqrt{-1} \pi \beta\left(X_{j}\right) I d \quad \forall 1 \leq j \leq p .
$$

Case: $p+1 \leq j \leq n$. First note that

$$
\begin{aligned}
\exp \left(-t X_{j}\right) \exp \left(x_{1} X_{1}\right) \cdots \exp \left(x_{n} X_{n}\right) \\
=\left[\prod_{k=1}^{j-1} \exp \left(\operatorname{Ad}\left(\exp \left(-t X_{j}\right)\left(x_{k} X_{k}\right)\right)\right)\right] \\
\quad \times \exp \left(\left(x_{j}-t\right) X_{j}\right) \exp \left(x_{j+1} X_{j+1}\right) \cdots \exp \left(x_{n} X_{n}\right),
\end{aligned}
$$

where

$$
\begin{aligned}
A d\left(\exp \left(-t X_{j}\right)\right)\left(x_{k} X_{k}\right) & =\left[\exp \left(-\operatorname{tad}\left(X_{j}\right)\right)\right]\left(x_{k} X_{k}\right) \\
& =x_{k} X_{k}-t x_{k} \sum_{l=1}^{j-1} P_{l}(k, t) X_{l} \quad \forall 1 \leq k \leq j-1,
\end{aligned}
$$

where $P_{l}(k, t)$ is a polynomial in the variable $t$. Moreover, the Campbell-Hausdorff formula in [4] allows us to write that

$$
\prod_{k=1}^{j-1} \exp \left(x_{k} X_{k}-t x_{k} \sum_{l=1}^{j-1} P_{l}(k, t) X_{l}\right)=\exp \left(\sum_{k=1}^{j-1} x_{k} X_{k}-t \sum_{l=1}^{j-1} q_{l}(j-1 ; t, x) X_{l}\right)
$$

where $q_{l}(j-1 ; t, x)$ is a polynomial in the variables $t$ and $x=\left(x_{1}, \cdots, x_{j-1}\right)$ such that $q_{l}(j-1 ; t, 0)=0$ for all $1 \leq l \leq j-1$. The idea is then to rewrite the right side of (4.14) as follows:

$$
\begin{aligned}
& \exp \left(\sum_{k=1}^{j-1} x_{k} X_{k}-t \sum_{l=1}^{j-1} q_{l}(j-1 ; t, x) X_{l}\right) \\
& =\exp \left(\sum_{k=1}^{j-2} x_{k} X_{k}-t \sum_{l=1}^{j-2} q_{l}(j-1 ; t, x) X_{l}+\left(x_{j-1}-t q_{j-1}(j-1 ; t, x)\right) X_{j-1}\right) \\
& =\exp \left(\sum_{k=1}^{j-2} x_{k} X_{k}-t \sum_{l=1}^{j-2} q_{l}(j-1 ; t, x) X_{l}+\left(x_{j-1}-t q_{j-1}(j-1 ; t, x)\right) X_{j-1}\right) \\
& \times \exp \left(-\left(x_{j-1}-t q_{j-1}(j-1 ; t, x)\right) X_{j-1}\right) \exp \left(\left(x_{j-1}-t q_{j-1}(j-1 ; t, x)\right) X_{j-1}\right) .
\end{aligned}
$$

Again the Campbell-Hausdorff formula implies that

$$
\begin{aligned}
& \exp \left(\sum_{k=1}^{j-1} x_{k} X_{k}-t \sum_{l=1}^{j-1} q_{l}(j-1 ; t, x) X_{l}\right) \\
& =\exp \left(\sum_{k=1}^{j-2} x_{k} X_{k}-t \sum_{l=1}^{j-2} q_{l}(j-2 ; t, x) X_{l}\right) \exp \left(\left(x_{j-1}-t q_{j-1}(j-1 ; t, x)\right) X_{j-1}\right)
\end{aligned}
$$


where $q_{l}(j-2 ; t, x)$ is a polynomial in the variables $t$ and $x=\left(x_{1}, \cdots, x_{j-1}\right)$, such that $q_{l}(j-2 ; t, 0)=0$. We apply the same process to

$$
\exp \left(\sum_{k=1}^{j-2} x_{k} X_{k}-t \sum_{j=1}^{j-2} q_{l}(j-2 ; t, x) X_{l}\right) .
$$

After $j-2$ steps, we obtain that

$$
\exp \left(\sum_{k=1}^{j-1} x_{k} X_{k}-t \sum_{l=1}^{j-1} q_{l}(j-1 ; t, x) X_{l}\right)=\prod_{k=1}^{j-1} \exp \left(\left(x_{k}-t q_{k}(k ; t, x)\right) X_{k}\right)
$$

where, for all $1 \leq k \leq j-1, q_{k}(k ; t, x)$ is a polynomial in the variables $t$ and $x=\left(x_{1}, \cdots, x_{j-1}\right)$ such that $q_{k}(k ; t, 0)=0$. Thus, we have

$$
\begin{aligned}
& {\left[L\left(X_{j}\right)\right]^{\sharp} f^{\sharp}\left(x_{1}, \cdots, x_{n}\right)} \\
& =\left.\frac{d}{d t} f^{\sharp}\left(x_{1}-t q_{1}(1 ; t, x), \cdots, x_{j-1}-t q_{j-1}(j-1 ; t, x), x_{j}-t, x_{j+1}, \cdots, x_{n}\right)\right|_{t=0} .
\end{aligned}
$$

If we put $q_{k}(x)=q_{k}(k ; 0, x), 1 \leq k \leq j-1$, we obtain the result using the $H$ covariance of $f$ in $C^{\infty}(G, H, \beta)$.

As we said before (Section 3), we view the symmetric algebra $S(\mathfrak{g})\left(\operatorname{resp} . S^{m}(\mathfrak{g})\right)$ of $\mathfrak{g}$ as the algebra $\mathbb{C}\left[\mathfrak{g}^{*}\right]$ of polynomials (resp. polynomials of degree $m$ ) on $\mathfrak{g}^{*}$. Denote by $S(\mathfrak{g})^{H}\left(\right.$ resp. $\left.S^{m}(\mathfrak{g})^{H}\right)$ its subalgebra of $H$-invariant polynomials on $\mathfrak{g}^{*}$ defined by

$$
\mathbb{C}\left[\mathfrak{g}^{*}\right]^{H}=\left\{P \in \mathbb{C}\left[\mathfrak{g}^{*}\right] \mid A d(h)(P)(l)=P(l) \forall h \in H \forall l \in \mathfrak{g}^{*}\right\} .
$$

It is clear that any polynomial on $\mathfrak{g}^{*}$ can be written as a finite sum of homogeneous polynomials. Then we have

$$
\forall m \in \mathbb{N} \forall Y \in H \quad \forall P \in S^{m}(\mathfrak{g}), \quad \operatorname{ad}(Y)(P) \in S^{m}(\mathfrak{g})
$$

so that

$$
S(\mathfrak{g})^{H}=\bigoplus_{m \geq 0} S^{m}(\mathfrak{g})^{H}
$$

On the other hand, using the basis $\left\{X_{1}, \cdots, X_{n}\right\}$ defined by (4.5), we write for multi-indexes in $\mathbb{N}^{n},(\nu, \alpha)=\left(\nu_{1}, \cdots, \nu_{p}, \alpha_{p+1}, \cdots, \alpha_{n}\right)$. Then, following the Poincaré-Birkhoff-Witt Theorem, any element of $\mathcal{U}(\mathfrak{g})$ can be written as

$$
\begin{aligned}
& \sum_{(\alpha, \nu) \in \mathbb{N}^{n-p} \times \mathbb{N}^{p}} a_{\nu, \alpha} X_{n}^{\alpha_{n}} \cdots X_{p+1}^{\alpha_{p+1}} X_{p}^{\nu_{p}} \cdots X_{1}^{\nu_{1}} \\
& \equiv \sum_{(\alpha, \nu) \in \mathbb{N}^{n-p} \times \mathbb{N}^{p}} a_{\nu, \alpha} X^{\alpha} X^{\nu} \text { with } a_{\nu, \alpha} \in \mathbb{C} .
\end{aligned}
$$

However, to avoid confusion between $\mathcal{U}(\mathfrak{g})$ and $S(\mathfrak{g})$, we shall use small letters for the basis of $\mathfrak{g}$ defined by (4.5) to write any polynomial on $\mathfrak{g}^{*}$ as

$$
\sum_{(\alpha, \nu) \in \mathbb{N}^{n-p} \times \mathbb{N}^{p}} a_{\nu, \alpha} x_{n}^{\alpha_{n}} \cdots x_{p+1}^{\alpha_{p+1}} x_{p}^{\nu_{p}} \cdots x_{1}^{\nu_{1}} \equiv \sum_{(\alpha, \nu) \in \mathbb{N}^{n-p} \times \mathbb{N}^{p}} a_{\nu, \alpha} x^{\alpha} x^{\nu} \text { with } a_{\nu, \alpha} \in \mathbb{C} .
$$


As usual, if $\lambda \in \mathbb{N}^{n}$ is a multi-index, we shall denote its length as the number $|\lambda|=\sum_{k=1}^{n} \lambda_{k}$; so that the degree of the element $\sum_{(\nu, \alpha) \in \mathbb{N}^{n}} a_{\nu, \alpha} X^{\alpha} X^{\nu}$ of $\mathcal{U}(\mathfrak{g})$ is the number $|\alpha|+|\nu|$. In the sequel, we shall denote by $\mathcal{U}_{m}(\mathfrak{g})$ the vector subspace of $\mathcal{U}(\mathfrak{g})$ of the elements with degree at most $m$.

Lemma 3. Let $G$ be a simply connected connected nilpotent Lie group. Assume $H$ is a commutative subgroup of $G$. Let $\mathcal{P}$ be an $H$-invariant homogeneous polynomial on $\mathfrak{g}^{*}$ such that $\mathcal{P}$ does not vanish identically on $\mathfrak{g}^{*}$. Then there exists a non-empy Zariski open subset $\mathcal{O}$ of $\mathfrak{h}^{*}$, such that for all $\beta$ in $\mathcal{O}$, we have $\left(L_{\beta} \circ \sigma\right)(\mathcal{P}) \neq 0$ in $\mathcal{D}(G, H, \beta)$, where $L_{\beta}$ is the isormorphism induced by (3.2).

Proof. Suppose that $\mathcal{P}$ is a homogeneous polynomial of degree $d$ which does not vanish identically on $\mathfrak{g}^{*}$. We can write as in (4.23):

$$
\mathcal{P}=\sum_{\substack{(\alpha, \nu) \in \mathbb{N} n-p \times \mathbb{N} p \\|\alpha|+|\nu|=d}} a_{\nu, \alpha} x^{\alpha} x^{\nu}
$$

with $a_{\nu, \alpha}$ in $\mathbb{C}$. Then applying the symmetrization map to $\mathcal{P}$, we get

$$
\sigma(\mathcal{P})=\sum_{\substack{(\alpha, \nu) \in \mathbb{N}^{n}-p \times \mathbb{N}^{p} \\|\alpha|+|\nu|=d}}\left(a_{\nu, \alpha} X^{\alpha} X^{\nu}+W_{\alpha, \nu}\right)
$$

where

$$
W_{\alpha, \nu}=\sum_{\substack{\left(\alpha^{\prime}, \nu^{\prime}\right) \in \mathbb{N}^{n-p} \times \mathbb{N}^{p} \\\left|\alpha^{\prime}\right|+\left|\nu^{\prime}\right|<|\alpha|+|\nu|}} b_{\nu^{\prime}, \alpha^{\prime}} X^{\alpha^{\prime}} X^{\nu^{\prime}} .
$$

Actually, we can rewrite (4.25) as follows:

$$
\sigma(\mathcal{P})=\sum_{\alpha \in \mathbb{N}^{n-p}} X^{\alpha}\left(\sum_{\substack{\nu \in \mathbb{N}^{p} \\|\nu|=d-|\alpha|}} a_{\alpha, \nu} X^{\nu}+\sum_{\substack{\nu^{\prime} \in \mathbb{N}^{p} \\\left|\nu^{\prime}\right|<d-|\alpha|}} b_{\alpha, \nu^{\prime}} X^{\nu^{\prime}}\right) .
$$

Let us define the polynomial $\mathcal{P}_{\alpha}$ on $\mathfrak{h}^{*}$ as

$$
\mathcal{P}_{\alpha}=\sum_{\substack{\nu \in \mathbb{N}^{p} \\|\nu|=d-|\alpha|}} a_{\alpha, \nu} x^{\nu}+\sum_{\substack{\nu^{\prime} \in \mathbb{N}^{p} \\\left|\nu^{\prime}\right|<d-|\alpha|}} b_{\alpha, \nu^{\prime}} x^{\nu^{\prime}}
$$

such that

$$
\left(L_{\beta} \circ \sigma\right)(\mathcal{P})=\sum_{\alpha \in \mathbb{N}^{n-p}} \mathcal{P}_{\alpha}(-2 \sqrt{-1} \pi \beta) X^{\alpha} .
$$

Define the subset $\mathcal{A}_{\mathcal{P}}$ of multi-indexes in $\mathbb{N}^{n-p}$ by

$$
\mathcal{A}_{\mathcal{P}}=\left\{\alpha \in \mathbb{N}^{n-p} \mid \mathcal{P}_{\alpha} \not \equiv 0\right\} .
$$

Since $\mathcal{P}$ does not vanish identically on $\mathfrak{g}^{*}$, there exists a multi-index $\alpha$ in $N^{n-p}$ such that $\mathcal{P}_{\alpha}$ does not vanish identically on $\mathfrak{h}^{*}$, so that the subset $\mathcal{A}_{\mathcal{P}}$ is not empty. Next define the variety $\mathcal{M}_{\mathcal{P}}$ of $\mathfrak{h}^{*}$ by

$$
\mathcal{M}_{\mathcal{P}}=\bigcap_{\alpha \in \mathcal{A}_{\mathcal{P}}}\left\{\mathcal{P}_{\alpha}=0\right\} .
$$

It is clear that $\mathcal{M}_{\mathcal{P}}$ is a non-empty Zariski closed subset of $\mathfrak{h}^{*}$ which differs from $\mathfrak{h}^{*}$. Then we define $\mathcal{O}_{\mathcal{P}}$ as the non-empty Zariski open subset of $\mathfrak{h}^{*}$ :

$$
\mathcal{O}_{\mathcal{P}}=\mathfrak{h}^{*} \backslash \mathcal{M}_{\mathcal{P}}
$$


On the other hand, for all linear forms $l$ in $\mathfrak{h}^{*}$, define the subset $\mathcal{A}_{\mathcal{P}, l}$ of $\mathcal{A}_{\mathcal{P}}$ by

$$
\mathcal{A}_{\mathcal{P}, l}=\left\{\alpha \in \mathcal{A}_{\mathcal{P}} \mid \mathcal{P}_{\alpha}(l) \neq 0\right\} .
$$

Note that if $l$ is in $\mathcal{O}_{\mathcal{P}}$, then $\mathcal{A}_{\mathcal{P}, l}$ is not empty.

Finally, fix $\beta$ in $\mathcal{O}_{\mathcal{P}}$. Let $\xi$ be an element of maximal length in $\mathcal{A}_{\mathcal{P}, \beta}$. We define a function $\phi_{\xi}$ in $C^{\infty}(G, H, \beta)$ as follows:

$\phi_{\xi}\left(\exp \left(t_{1} X_{1}\right) \cdots \exp \left(t_{n} X_{n}\right)\right)=\chi_{\beta}\left(\exp \left(t_{1} X_{1}\right) \cdots \exp \left(t_{p} X_{p}\right)\right)\left(-t_{p+1}\right)^{\xi_{p+1}} \cdots\left(-t_{n}\right)^{\xi_{n}}$.

$\phi_{\xi}$ is a homogeneous function of degree $|\xi|$ in the variables $t_{p+1}, \cdots, t_{n}$. Using Lemma 2 together with (4.29), we obtain that

$$
\left[\left(L_{\beta} \circ \sigma\right)(\mathcal{P})\right]^{\sharp}\left(\phi_{\xi}^{\sharp}\right)(0)=\mathcal{P}_{\xi}(-2 \sqrt{-1} \pi \beta) t_{p+1} ! \cdots t_{n} ! .
$$

We have $\left[\left(L_{\beta} \circ \sigma\right)(\mathcal{P})\right]^{\sharp}\left(\phi_{\xi}^{\sharp}\right) \neq 0$. Hence $\left[\left(L_{\beta} \circ \sigma\right)(\mathcal{P})\right]\left(\phi_{\xi}\right) \neq 0$. This shows that $\left(L_{\beta} \circ \sigma\right)(\mathcal{P}) \neq 0$.

\subsection{A second theorem.}

Theorem 2. Let $G$ be a simply connected connected real nilpotent Lie group with Lie algebra $\mathfrak{g}, H$ a connected closed commutative subgroup of $G$ with Lie algebra $\mathfrak{h}$. Consider a weak Malcev basis passing through $\mathfrak{h}$. Then, if $\mathfrak{h}+\mathfrak{g}(l)$ is not lagrangian in $\mathfrak{g}$ for generic $l$ in $\Omega_{G, H, \beta}$, the algebra $\mathcal{D}(G, H, \beta)$ is not commutative, for all $\beta$ in a non-empty Zariski open subset of $\mathfrak{h}^{*}$.

Proof. Let $\mathfrak{g}_{0}$ be an ideal of codimension one of $\mathfrak{g}$ containing $\mathfrak{h}$ and $G_{0}=\exp \left(\mathfrak{g}_{0}\right)$. One can suppose that $\tau_{0}=\operatorname{In} d_{H}^{G_{0}} \chi_{\beta}$ is of finite multiplicities. Under the notation of Section 2, we take $V=\mathfrak{g}^{*}$. So it is clear that $V^{\text {gene }} \cap \Omega_{G, H, \beta} \neq \emptyset$ for almost all $\beta$ in $\mathfrak{h}^{*}$. Under the assumptions of the Theorem 2, the Pukanszky parametrization of the $H$-orbits in $\mathfrak{g}^{*}$, outlined in Section 2, gives a non-zero $H$-invariant polynomial $\mathcal{P}$ on $\mathfrak{g}^{*}$ such that $\mathcal{P} \notin S\left(\mathfrak{g}_{0}\right)$. Moreover, using (4.20) - (4.21), one can suppose that $\mathcal{P}$ is homogeneous. Then, from Lemma $3, \sigma(\mathcal{P}) \notin \mathcal{U}\left(\mathfrak{g}_{0}\right)+\mathcal{U}(\mathfrak{g}) \mathfrak{a}_{\beta}$ and $\left(L_{\beta} \circ \sigma\right)(\mathcal{P})$ is a non-zero element of $\mathcal{D}(G, H, \beta)$, for all $\beta$ in $\mathcal{O}_{\mathcal{P}}$, as defined by (4.32). Thus, we apply Theorem 1 to get an element $T$ of $\mathcal{U}\left(\mathfrak{g}_{0}, \mathfrak{h}, \beta\right)$ such that $\left[\left(L_{\beta} \circ \sigma\right)(\mathcal{P}), L_{\beta}(T)\right] \neq 0$ in $\mathcal{D}(G, H, \beta)$.

\subsection{The case where $\mathfrak{h}$ is an ideal of $\mathfrak{g}$.}

Corollary 2. Let $G$ be a simply connected connected real nilpotent Lie group with Lie algebra $\mathfrak{g}$ and $H$ a connected closed normal subgroup of $G$ with Lie algebra $\mathfrak{h}$. Then, for almost all $\beta$ in $\mathfrak{h}^{*}$ satisfying $\beta([\mathfrak{h}, \mathfrak{h}])=\{0\}$, the assertions $(i),($ ii) and (iii) of Section 2 are equivalent.

Proof. $(i) \Rightarrow(i i)$ : Under the notation of Section 2, we take $V=[\mathfrak{h}, \mathfrak{h}]^{\perp}$ and we choose $\beta$ in the fundamental layer of $V$ to apply Theorem 2 .

For $(i i) \Rightarrow(i i i)$ and $(i i i) \Rightarrow(i)$ see the end of Section 2 .

\section{Characterization of $\mathcal{D}(G, H, \beta)$ in terms of the algebra of $A d^{*}(H)$-InVARIant RATIONAL FUnCtions on $\Omega_{G, H, \beta}$}

We shall denote by $\pi_{l}$ the representation associated to $l \in \Omega_{G, H, \beta} / H$ by the Kirillov map and by $d \tilde{\mu}$ the image on $\Omega_{G, H, \beta} / H$ of the Lebesgue measure on $\Omega_{G, H, \beta}$. 
If $\phi=\int_{\Omega_{G, H, \beta} / H}^{\oplus} \phi_{\pi_{l}} d \tilde{\mu}(l)$, then

$$
D \phi=\int_{\Omega_{G, H, \beta} / H}^{\oplus} \Theta^{\tau}(D, l) \phi_{\pi_{l}} d \tilde{\mu}(l) \forall D \in \mathcal{D}(G, H, \beta)
$$

where $\Theta^{\tau}(D,$.$) belongs to \mathbb{C}\left(\Omega_{G, H, \beta}\right)^{H}$, the algebra of $A d^{*}(H)$-invariant rational functions on $\Omega_{G, H, \beta}$. The application $\Theta^{\tau}: \mathcal{D}(G, H, \beta) \rightarrow \mathbb{C}\left(\Omega_{G, H, \beta}\right)^{H}$ is an isomorphism between $\mathcal{D}(G, H, \beta)$ and a subalgebra of $\mathbb{C}\left(\Omega_{G, H, \beta}\right)^{H}$. Actually Fujiwara proved that if there exists a common polarization for almost all linear forms on $\mathfrak{g}$ whose restriction to $\mathfrak{h}$ is $\beta$ or if $\mathfrak{h}$ is 1-dimensional, then $\Theta^{\tau}$ is an isomorphism between $\mathcal{D}(G, H, \beta)$ and $\mathbb{C}\left[\Omega_{G, H, \beta}\right]^{H}$, the algebra of $A d^{*}(H)$-invariant polynomials on $\Omega_{G, H, \beta} 8$. This gives a partial answer to a question of Corwin and Greenleaf [5], also asked by Duflo (Problème 3 of [7]) in a more general context.

In the particular cases studied above, we have

Corollary 3. Let $G$ be a connected simply connected real nilpotent Lie group with Lie algebra $\mathfrak{g}$ and $H$ a connected closed subgroup of $G$ with Lie algebra $\mathfrak{h}$. The following assertions $(a)$ and $(b)$ are equivalent:

- for all $\beta$ in $\mathfrak{h}^{*}$ satisfying $\beta([\mathfrak{h}, \mathfrak{h}])=\{0\}$ when $(\mathfrak{g}, \mathfrak{h})$ is a reductive pair,

- for almost all $\beta$ in $\mathfrak{h}^{*}$ satisfying $\beta([\mathfrak{h}, \mathfrak{h}])=\{0\}$ when $\mathfrak{h}$ is commutative or $\mathfrak{h}$ is an ideal in $\mathfrak{g}$.

(a) $\mathcal{D}(G, H, \beta)$ is a commutative algebra.

(b) $\mathcal{D}(G, H, \beta)$ is isomorphic, via $\Theta^{\tau}$, to a subalgebra of $\mathbb{C}\left(\Omega_{G, H, \beta}\right)^{H}$.

Proof. $(a) \Rightarrow(b)$ : From Theorem 2 and Corollaries 1 and 2 if $\mathcal{D}(G, H, \beta)$ is commutative, then $\tau$ is of finite multiplicities, so that the results of [5] apply.

$(b) \Rightarrow(a)$ is obvious.

Remark 5. Note that in the particular reductive case where $H$ is one-dimensional (Remark 3 ) or if $H$ is a normal subgroup of $G$ (Corollary 2), then from 8 , the image of $\mathcal{D}(G, H, \beta)$ under $\Theta^{\tau}$ is, actually, the algebra $\mathbb{C}\left[\Omega_{G, H, \beta}\right]^{H}$ of $A d^{*}(H)$-invariant polynomials on $\Omega_{G, H, \beta}$.

\section{EXAMPLES}

In the following examples $\mathfrak{g}$ will be the real nilpotent Lie algebra of dimension 7 generated by the vectors $\left\{X_{i}, 1 \leq i \leq 7\right\}$ with the following non-zero brackets:

$\left[X_{1}, X_{3}\right]=X_{2},\left[X_{1}, X_{4}\right]=X_{3},\left[X_{1}, X_{5}\right]=X_{4},\left[X_{1}, X_{7}\right]=X_{6},\left[X_{4}, X_{5}\right]=X_{6}$,

$\left[X_{5}, X_{6}\right]=X_{2}$ and $\left[X_{4}, X_{7}\right]=-X_{2}$.

Moreover, in the following examples, $\tau=\operatorname{Ind} d_{H}^{G} \chi_{\beta}$ is of infinite multiplicities.

Example 1. Take $\mathfrak{h}=\mathbb{R} X_{1}$ and $\beta=\xi_{1} X_{1}^{*}$. Put $l=\sum_{i=1}^{7} \xi_{i} X_{i}^{*}$ with $\xi_{2} \neq 0$. Corollary 1 and Remark 3 apply in this situation. We take the Malcev basis ordered in the following way: $X_{1}, X_{2}, X_{3}, X_{4}, X_{6}, X_{5}$ and $X_{7}$. This defines a Jordan-Hölder sequence of subalgebras of $\mathfrak{g}$. It happens that

$$
A d^{\star}\left(\exp \left(-t X_{1}\right)\right)\left(\sum_{i=1}^{7} \xi_{i} X_{i}^{\star}\right)=\sum_{i=1}^{7} \xi_{i}(t) X_{i}^{\star}
$$

with

$$
\begin{aligned}
& \xi_{1}(t)=\xi_{1}, \quad \xi_{2}(t)=\xi_{2}, \quad \xi_{3}(t)=\xi_{3}+t \xi_{2}, \\
& \xi_{4}(t)=\xi_{4}+t \xi_{3}+\frac{1}{2} t^{2} \xi_{2}, \quad \xi_{6}(t)=\xi_{6}, \\
& \xi_{5}(t)=\xi_{5}+t \xi_{4}+\frac{1}{2} t^{2} \xi_{3}+\frac{1}{6} t^{3} \xi_{2}, \quad \xi_{7}(t)=\xi_{7}+t \xi_{6} .
\end{aligned}
$$


We parametrize the $H$-orbits by $u=\xi_{3}+t \xi_{2}$. The orbit of $\ell$ is of dimension 1 and is exactly the set $\left\{\ell(u)=\sum r_{i}(\ell, u) X_{i}^{\star}, u \in \mathbb{R}\right\}$ where

$r_{1}(\ell, u)=\xi_{1}, \quad r_{2}(\ell, u)=\xi_{2}$,

$r_{3}(\ell, u)=u$, at this step, dimension of orbits passes from 0 to 1

$r_{4}(\ell, u)=\frac{2 \xi_{2} \xi_{4}-\xi_{3}^{2}}{2 \xi_{2}}+\frac{1}{2 \xi_{2}} u^{2}, \quad r_{6}(\ell, u)=\xi_{6}$,

$r_{5}(\ell, u)=\frac{1}{6 \xi_{2}^{2}} u^{3}+\frac{2 \xi_{2} \xi_{4}-\xi_{3}^{2}}{2 \xi_{2}^{2}} u+\frac{\xi_{3}^{3}+3 \xi_{2}^{2} \xi_{5}-3 \xi_{2} \xi_{3} \xi_{4}}{3 \xi_{2}^{2}}, \quad r_{7}(\ell, u)=\frac{\xi_{6}}{\xi_{2}} u+\frac{\xi_{2} \xi_{7}-\xi_{3} \xi_{6}}{\xi_{2}}$.

Thus, this gives us rational functions and then $H$-invariant polynomial functions that are written in terms of the variables $\xi_{i}$. The elements of $\mathcal{U}(\mathfrak{g}, \mathfrak{h}, \beta)$ obtained by symmetrization are: $X_{1}, X_{2}, 2 X_{2} X_{4}-X_{3}^{2}, X_{6}, X_{3}^{3}+3 X_{2}^{2} X_{5}-3 X_{2} X_{3} X_{4}$ and $X_{2} X_{7}-X_{3} X_{6}$.

We have $\left[X_{3}^{3}+3 X_{2}^{2} X_{5}-3 X_{2} X_{3} X_{4}, X_{6}\right]=3 X_{2}^{3} \notin \mathcal{U}(\mathfrak{g}) \mathfrak{a}_{\beta}$ and

$\left[X_{2} X_{7}-X_{3} X_{6}, 2 X_{2} X_{4}-X_{3}^{2}\right]=2 X_{2}^{3} \notin \mathcal{U}(\mathfrak{g}) \mathfrak{a}_{\beta}$. The left action of these elements on $C^{\infty}(G, H, \beta)$ gives elements of the algebra $\mathcal{D}(G, H, \beta)$, which is not commutative.

Example 2. Put $\mathfrak{h}=\mathbb{R} X_{1} \oplus \mathbb{R} X_{6}$ with $\beta=\xi_{1} X_{1}^{*}+\xi_{6} X_{6}^{*}$ where $\xi_{6} \neq 0$. Since $\mathfrak{h}$ is commutative, we apply Theorem 2 . The condition $\xi_{6} \neq 0$ ensures the coincidence of the fundamental and generic layers. Analogous calculations as those of Example 1 give the following elements of $\mathcal{U}(\mathfrak{g}, \mathfrak{h}, \beta)$ whose images under $L$ belong to $\mathcal{D}(G, H, \beta)$ : $X_{6}, X_{1}, X_{2}, X_{2} X_{7}-X_{3} X_{6}$ and $2 X_{4} X_{6}^{2}-2 X_{3} X_{6} X_{7}+X_{2} X_{7}^{2}$.

As in the previous example, the algebra $\mathcal{D}(G, H, \beta)$ is not commutative, since $\left[X_{2} X_{7}-X_{3} X_{6}, 2 X_{4} X_{6}^{2}-2 X_{3} X_{6} X_{7}+X_{2} X_{7}^{2}\right]=2 X_{2}^{2} X_{6}^{2} \notin \mathcal{U}(\mathfrak{g}) \mathfrak{a}_{\beta}$.

Example 3. Take $\mathfrak{h}=\mathbb{R} X_{1} \oplus \mathbb{R} X_{6}$ with $\beta=\xi_{1} X_{1}^{*}+\xi_{6} X_{6}^{*}$ where $\xi_{6}=0$. Analogous calculations as those of Example 1 give the following elements of $\mathcal{U}(\mathfrak{g}, \mathfrak{h}, \beta)$ : $X_{6}, X_{1}, X_{7}, X_{2}$ and $2 X_{2} X_{4}-X_{3}^{2}$.

Since $\left[2 X_{2} X_{4}-X_{3}^{2}, X_{7}\right]=-2 X_{2}^{2} \notin \mathcal{U}(\mathfrak{g}) \mathfrak{a}_{\beta}$, the algebra $\mathcal{D}(G, H, \beta)$ is not commutative. Here it is interesting to note that our situation is degenerated. However, we observe that in this example the previous constructions give a non-commutative family of elements in $\mathcal{D}(G, H, \beta)$.

\section{REFERENCES}

1. A. Baklouti and J. Ludwig, Invariant differential operators on certain nilpotent homogeneous spaces. To appear in Monatshefte für Mathematik.

2. P. Bernat, N. Conze, M. Duflo, M. Lévy-Nahas, M. Rais, P. Renouard, M. Vergne, Représentations des groupes de Lie résolubles. Monographies de la Société Mathématique de France, No 4, Dunod, Paris, 1972. MR 56:3183

3. L. Corwin and F. P. Greenleaf, A canonical approach to multiplicity formulas for induced and restricted representations of nilpotent Lie groups. Comm. Pure Appl. Math., 41, 1988. MR 90b:22011b

4. L. Corwin and F. P. Greenleaf, Representations of nilpotent Lie groups and their applications, Part I. Cambridge Studies in Adv. Math., No 18, Cambridge Univ. Press, 1989. MR 92b:22007

5. L. Corwin and F. P. Greenleaf, Commutativity of invariant differential operators on nilpotent homogeneous spaces with finite multiplicity. Comm. Pure Appl. Math., 45, 1992. MR 93i:22010

6. L. Corwin and F. P. Greenleaf, Spectral Decomposition of Invariant Differential Operators on Certain Nilpotent Homogeneous Spaces. J. Funct. Analysis, 108, 1992. MR 93j:22009

7. M. Duflo, Open Problems in Representation Theory of Lie groups. Conference on "Analysis on homogeneous spaces", Katata, Japan, 1986.

8. H. Fujiwara, Sur la conjecture de Corwin-Greenleaf. J. of Lie Theory, 7, 1997.

9. F. P. Greenleaf, Harmonic analysis on nilpotent homogeneous spaces. Contemporary Mathematics, 177, 1994. MR 96e:22025 
10. F. P. Greenleaf, Geometry of coadjoint orbits and noncommutativity of invariant differential operators on nilpotent homogeneous spaces. Comm. Pure Appl. Math., 53, 2000. CMP 2000:15

11. R. Lipsman, Orbital parameters for induced and restricted representations. Trans. Amer. Math. Soc., 313, 1989. MR 90a:22008

12. L. Pukanszky, Leçons sur les représentations des groupes. Monographies de la Société Mathématique de France, No 2, Dunod, Paris, 1967. MR 36:311

Faculté de Technologie À Kyushu, Université de Kinki, Iizuka 820-8555, Japon

E-mail address: fujiwara@fuk.kindai.ac.jp

Equipe Modal'X, Université Paris X, 200 Avenue de la République, 92001 Nanterre, FrANCE

Equipe de Théorie des Groupes, Représentations et Applications, Institut de Mathématiques de Jussieu, Université Paris ViI, 2 Place Jussieu, 75251 Paris Cedex 05, France

E-mail address: glion@math.jussieu.fr

Equipe Modal'X, Université Paris X, 200 Avenue de la République, 92001 Nanterre, FRANCE

Equipe de Théorie des Groupes, Représentations et Applications, Institut de Mathématiques de Jussieu, Université Paris Vit, 2 Place Jussieu, 75251 Paris Cedex 05, France

Current address, from 01/08/2000 to 31/05/2002: Department of Mathematics, Oklahoma State University, Stillwater, Oklahoma 74078-1058

E-mail address: smehdi@math.okstate.edu 